\title{
Optimizing Information Dissemination in Large Scale Mobile Peer- to-Peer Networks using Context-based Grouping
}

\author{
Ansar-Ul-Haque Yasar, Yves Vanrompay, Davy Preuveneers and Yolande Berbers
}

\begin{abstract}
Nowadays novel embedded computing devices enable vehicles to form large scale mobile peer-to-peer networks in which they can assist each other to improve their driving experience. Therefore context-aware communication is considered to be vital for inducing inter-vehicular intelligence between groups of vehicles with similar interests. However, traditional network addressing schemes are not well suited for group-based communication in large scale vehicular networks. The classical network paradigms of multicasting and broadcasting to define groups are too limited. First, there is no way to optimize network traffic based on the contextual characteristics of the nodes. Second, the groups of nodes are highly dynamic with vehicles randomly joining and leaving multiple groups. We propose an information dissemination approach based on context grouping in which only relevant information is shared among nodes. We evaluate our approach in a large scale vehicular network where groups are formed based on the location and shared interests of the nodes. The experiments show that by inducing our context-based grouping mechanism we can significantly eliminate irrelevant information and reduce overall network traffic in a scalable way.
\end{abstract}

\section{INTRODUCTION}

$I^{2}$ NTELLIGENT telematic application development is a research area that has gained a lot of attention from the research community $[1,2,16,17,18]$. Application areas include emergency message transmission, collision avoidance, congestion monitoring and intelligent parking space allocation. In this paper, we propose a novel way of vehicles in a large scale network to disseminate relevant context-aware information to each other in a peer-to-peer fashion so that they can make use of this information in order to perform context sensitive tasks. Such context-aware coordinated communication will enable the vehicles to share information with each other while not being flooded with irrelevant information and to use information which they cannot sense themselves directly.

Optimizing information sharing and retrieval between nodes in large scale dynamic networks is not straightforward, as processing the information to check

Manuscript received April 9, 2010; revised July 8, 2010.

A. Y. is with the Department of Computer Science, Katholieke Universiteit Leuven, Celestijnenlaan 200A bus 2402, B-3001 Heverlee, Belgium. (Corresponding Author) (Phone: +32-16-327535, Fax: +32-16327996, Email: ansarulhaque.yasar@cs.kuleuven.be).

Y. V., D. P. and Y. B. are with the Department of Computer Science, Katholieke Universiteit Leuven, Celestijnenlaan 200A bus 2402, B-3001 Heverlee, Belgium. (\{yves.vanrompay, davy.preuveneers and yolande.berbers\}@cs.kuleuven.be). where and when it is relevant and making sure it is routed in a peer-to-peer fashion to the right destination is a challenge. The classical network paradigms of multicasting and broadcasting to define groups are too limited. First, there is no way to optimize network traffic based on the contextual characteristics of the nodes. Second, the groups of nodes are highly dynamic with no fixed set of nodes per group. In current networks there is only limited sharing of information. For example, the same traffic jam information is broadcasted over the radio for every vehicle on the road not taking into account whether that information is relevant. Moreover, the 3G network technologies available for use via smart-phones are relatively expensive and impractical to be used inside the cars.

In this paper we address these issues by proposing a context-based grouping mechanism to optimize information dissemination using relevance backpropagation, a feedbackbased algorithm for identifying useful information, for information sharing. The relevance backpropagation algorithm was first proposed in [15]. Further information on how it is applied in vehicular networks can be found in [14]. Our context-based grouping mechanism allows formation of the groups using common contextual characteristics like location, direction, interests etc. For example, in convoy driving a lead car equipped with communication capabilities controls a group of cars without any human intervention to jointly reach a destination. These cars form a group based on their location and shared interests. Our approach also presents a simpler version of privacy mechanism as the information is distributed only to the interested groups and the members of that particular group.

The rest of the paper is organized as follows: In section II, we present our motivating scenario with a set of requirements. In section III, we describe our large scale peer-to-peer context dissemination middleware in detail. Experimental evaluation and results are presented in section IV. In section $\mathrm{V}$, we discuss some related work in comparison to our proposed scheme. Last but not the least we present our conclusion with some ideas for future work in section VI.

\section{MOTIVATING SCENARIO AND REQUIREMENTS FOR CONTEXT-BASED GROUPING}

In this section, we describe a motivating scenario in the area of vehicular networks as an example of large scale mobile peer-to-peer networks. We also derive a set of requirements that are considered vital for optimizing information dissemination in a large scale vehicular network. 


\section{A. Information sharing between vehicles and other nodes in a metropolitan city}

Nowadays, most of the new vehicles have an embedded Global Positioning System (GPS) device to assist drivers while going from one location to another. Let us take a typical case of Brussels city during the rush hours when there are thousands of vehicles on the roads. Several of these vehicles are interested in traffic information near and towards their destination. Moreover, some vehicles might be looking for the cheapest gas stations in the neighborhood. Such detailed and evolving information is hardly managed and never disseminated to the interested nodes in the vicinity. Even the installed GPS is of no use in this situation since it has no specific information like fuel prices.

By taking into consideration the kind of information vehicles share with other nodes in the network, we can easily identify various groups of vehicles and nodes with similar interests about a particular kind of information at a given time and location. For example, in a large city there are a large number of vehicles interested in general traffic information and a sub-set of them interested in information about parking spots. In this case we have two larger groups of vehicles one interested in traffic and the other in parking information within a certain location.

Within these two large interest groups there can also be sub-interests. For example, some vehicles are interested to park in Brussels city near the central station on a Sunday at $6.30 \mathrm{pm}$ whereas other cars may be interested in a long term parking with a maximum parking charge of $3.5 \mathrm{EUR} /$ day. Traditionally the parking information is displayed on electronic boards within the city for different parking spot. In some cases when a particular vehicle finds a vacant parking space and reaches that parking space it is usually occupied by another vehicle as the information about free parking space was either too old or the information changed on the electronic board as soon as the vehicle passed by it.

Different types of interactions in this scenario either in the form of a query or a message are listed as under:

1. Is there a free parking spot within $500 \mathrm{~m}$ ?

2. How much is the parking fee after $6 \mathrm{pm}$ ?

3. What are the opening hours?

4. Are there any gas stations that can serve hybrid cars?

5. Are there parking spots charging less than 1 EUR/hour?

6. Inform other cars interested in a parking spot about a free space.

The questions 2 and 5 are very similar having a shared interest in a parking spot. The vehicles sending such queries can form a group and share the information about parking spots with each other. Thus using context-based grouping the network traffic can be significantly reduced.
B. Requirements for optimizing information dissemination in a large scale mobile peer-to-peer network using context-based grouping

In order to optimize context-aware information dissemination in a large scale peer-to-peer mobile network using context-based grouping we start with establishing a set of requirements to support such communication.

1) Location and direction-aware delivery of relevant information

It is always desirable to know the exact location of an incident for context-aware applications e.g. in case of an incident on the road the authorities should be notified about the exact location to react fast and all the vehicles only travelling towards that incident should be informed as that information will be irrelevant for vehicles moving away from the incident. So a context-aware application should also be able to sense, manipulate and disseminate relevant context information at the right location and direction only to the interested nodes.

2) On-time delivery of information

It is quite critical that the information being disseminated in a large scale network between nodes or group of nodes reaches the destination on-time. Timeliness of information is a special kind of property that that uses time as relevance criteria for information sharing. For example, information about a free parking spot might not be relevant for interested vehicles if arrive 30 mins after being disseminated by the car having left the parking spot.

3) Optimize communication overhead and delivery efficiency

It is also quite important to be able to quantify how much data that is being transmitted over the network is actually used per network peer and by the network as a whole on an application basis. In our grouping mechanism, information is only sent to a sub-set of the vehicles. This quantification will help to properly analyze, improve and compare various information sharing algorithms and communication protocols based on the parameters like filtering, communication overhead and routing efficiency. In this paper we try to quantify our optimization mechanism using context-based grouping which is explained later in the paper.

\section{A LARGE SCALE PEER-TO-PEER CONTEXT DISSEMINATION MIDDLEWARE}

In, Fig. 1, we can see the architectural view for optimizing information dissemination in large scale networks using grouping. In this figure we have three separate layers namely application layer, context layer and physical layer. The application layer consists of all the applications like parking application, the emergency response team application, etc. having specialized interests. In the middle, we have a very important layer which contains our middleware for optimizing information dissemination by forming groups sharing some common contextual characteristics. On the physical layer all the communication is being performed between the groups and nodes in a network according to the relevance of information received from the context layer. 
The main idea behind our grouping mechanism is to reduce irrelevant communication between nodes in a large scale peer-to-peer network. The nodes are virtually separated into groups on some common observable context characteristics like same location, direction, interests, velocity, etc. We will discuss about groups formation in detail later in this section.

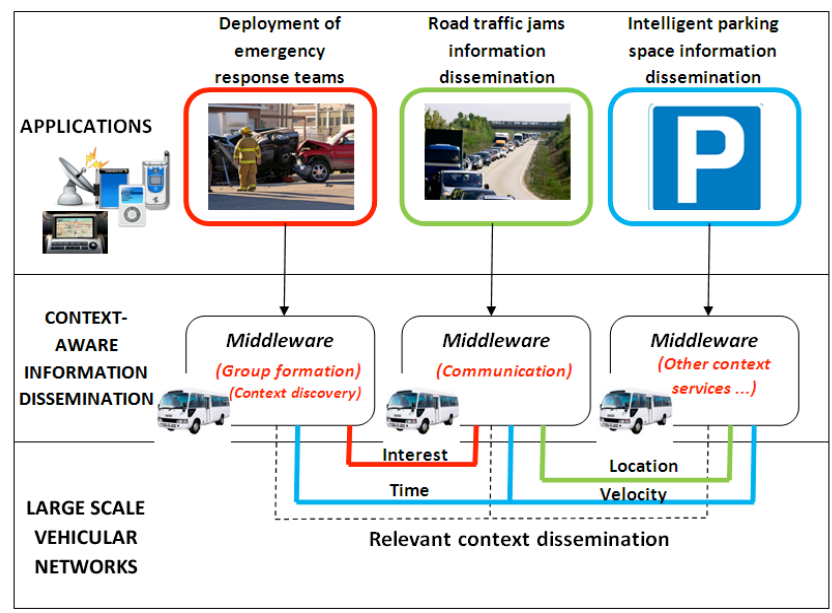

Fig. 1. Architecture for optimizing information dissemination in large scale networks.

\section{A. Context modeling for group-based communication in} a large scale peer-to-peer network

The proposed grouping mechanism defines groups based on shared context information like location or interests. Also, the information that is disseminated within groups involves context too. Thus it is important to be able to model context information as a generalization of semantic concepts. A context ontology provides a formal definition of context elements, their representation and semantics. In this work we make use of an ontology which contains specifications of context artifacts (such as interests) and their relations. This leads to a hierarchy of general and more specific concepts. For example, in the ontology "traffic information" is a general interest, and "traffic on highway $\mathrm{x}$ " is a sub-interest related to "traffic information". The concepts defined in the ontology (with their different granularity) correspond to the set of possible groups and subgroups in each location. In Fig. 2, we illustrate an interest by multiple inheritance in traffic jams on E314. Thus the scope is defined at the very top level, the interests and $s u b$ interests are the lower levels of our context ontology. The ontology also acts as a repository of existing interests for the nodes in the network.

\section{B. Context-aware groups formation}

The context-aware group formation is performed by the context layer in our middleware. The generic criterion for group formation is: $\mathrm{G}_{\mathrm{D}}=<\mathrm{C}_{\mathrm{D}}, \mathrm{I}_{\mathrm{D}}, \mathrm{SI}_{\mathrm{D}}>$, where $\mathrm{G}_{\mathrm{D}}$ is the group, $\mathrm{C}_{\mathrm{D}}$ is the criteria set like location (e.g. E314 or A2) for group formation, $I_{D}$ is the set of contextual information interests (e.g. traffic or parking information) and $\mathrm{SI}_{D}$ is the set of sub-interests (e.g. traffic jams or accidents). The application determines the criteria, interest and sub-interest set making sure that the groups are formed based on very specific interests and only relevant information can disseminate between the nodes inside a group. This concept also ensures privacy in a very basic manner on a need-toknow basis by restricting the flow of irrelevant information.

In Fig. 3 we can see two groups of traffic where one is interested in traffic jams on E314 and the other one interested in accident information on A2. Both these group are the part of a bigger traffic group in general. In this figure, node 3 is part of a context group on E314 and A2 interested in traffic jams and accident information respectively.

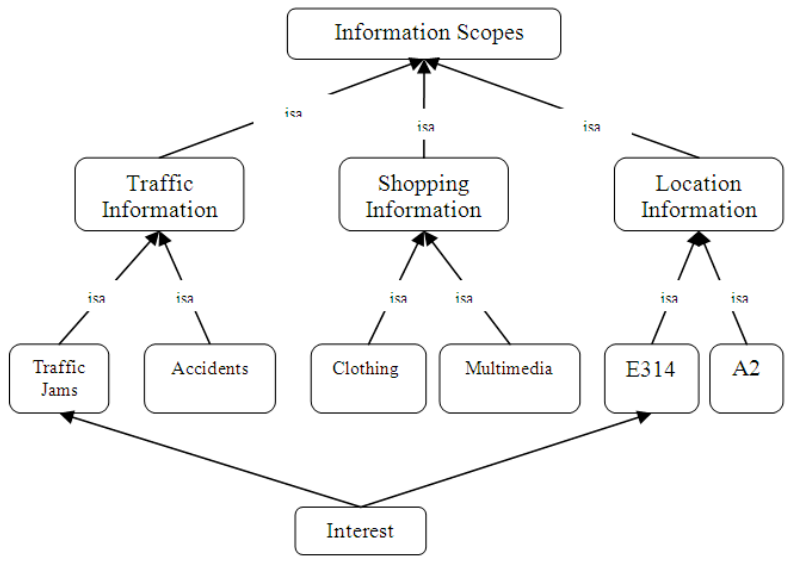

Fig. 2. Example ontology for group formation and context dissemination.

As in our context-based grouping mechanism the groups have nodes based on the common interests they share as derived from the application. So our mechanism can be compared to a generic decentralized publish / subscribe system where the peers can subscribe and publish only relevant information.

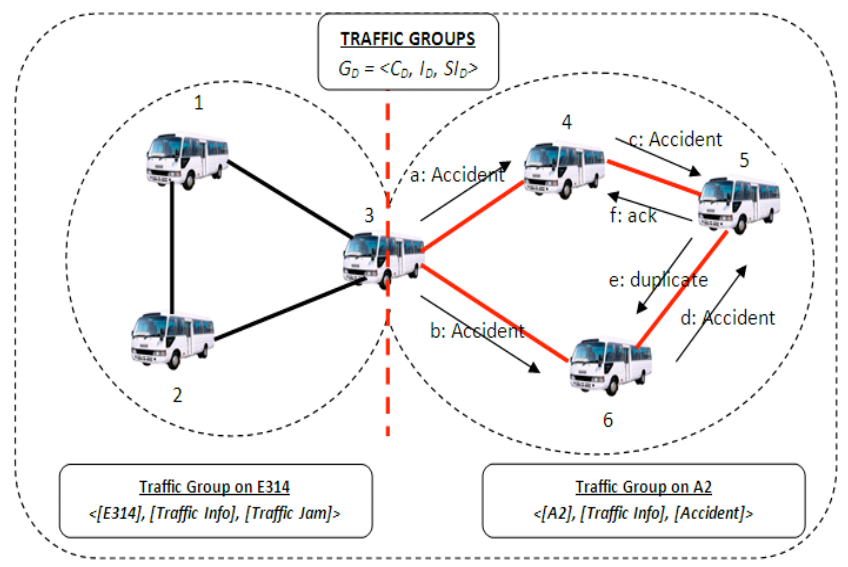

Fig. 3. Example of the context group formation based on common interests.

\section{Relevance backpropagation}

It is crucial that only relevant information is shared between the nodes in a large scale mobile peer-to-peer network. Otherwise would be flooded with information they 
cannot use saturating communication channels leaving no bandwidth to share information they really need. Initially the nodes are unaware of the type of relevant common context information they could share or receive. In order to establish an intelligent mechanism where nodes can share information, form groups with common interests and do context-sensitive tasks in a network, we need feedback from all the participating nodes. In order to achieve the objectives mentioned earlier, we introduce a special kind of an additional filter mechanism using relevance backpropagation to be used for inter and intra-group communication. More details on basic relevance backpropagation algorithm can be found in $[14,15]$.

We show in Fig. 3 that node 3 has information about an accident on A2 that is also of interest for nodes 4,5 and 6. Node 3 will forward to its immediate neighboring nodes, which in this case are 4 and 6 . They will forward the same relevant information to node 5 . Node 5 sends a feedback message to node 6 that it already received this information from another path. Node 4 will receive an acknowledgement from node 5 that it received useful information. The feedback is finally propagated back to node 3 .

Our relevance backpropagation algorithm which relies on feedback of neighboring nodes to reduce the number of peers to forward the information to as explained in the example. The information is initially forwarded to the adjacent nodes unless a maximum number of hops or other contextual criteria is reached. Each forwarding node reduces the hop counter, adds its identification and marks the message relevancy tag if the information is relevant for its purpose. The feedback technique is based on context information like position, velocity, direction, time-to-live, interest etc. that decides whether the data that was received is relevant or not and also helps determine the information relevancy on the intermediate nodes. The feedback to the delivering node is initiated if the context information is relevant, irrelevant, unused or duplicate information is received reducing the information dissemination only to the interested nodes. As the context information can be provided by the application itself the routing of the information is adapted accordingly and perhaps different for various applications. So the network recalibrates itself if a new node sends an arrival beacon or an old node no longer transmits the feedback information. In this mechanism the goal is to efficiently filter and route the relevant information as close to the source as possible in a dynamic network. Our algorithm is a best-effort algorithm which adapts itself according to the network configuration.

\section{Discovery, joining and updating of context-aware groups}

In this paper our focus is to present an implicit grouping mechanism for optimizing information dissemination in a large scale network. We divide the total area of the network into smaller sub-locations in the form of a grid based on certain physical characteristics of the network like spatial coverage. In our mechanism each node is a part of a virtual group with the same interests even if there is no other member present as shown in Fig. 4.

When the nodes move from one location to another they send a query to its neighboring nodes through plain broadcasting. The query contains the information about the node and its interest in general. Each of the node in that location query for the same information from nodes. A message to join the group is propagated back to the querying node only if there is a node or a number of nodes in a group with the same major interests. The node then can broadcast a groupLeave message to its current group, sends a groupJoin message to join the group at the next location and an update is performed at the level of the groups. A node can also join two or more groups at a particular location having common interests or sub-interests.



Fig. 4. Nodes joining and leaving groups in a large metropolitan city virtually divided into a grid of smaller locations.

Although the above example as shown in Fig. 4 is 2dimensional, our approach generalizes to n-dimensional criteria set in which groups are defined by n context types.

Our context-based grouping mechanism acts as a filtering technique to guarantee the relevance of information. In our mechanism we have a special kind of a filter inside a group where we drop irrelevant information by matching the subinterests of each node inside a group. For example, a car interested in traffic information on highway E40 and E19 joins a traffic information group on highway E314 but may only communicate if it can find another group or node with matching sub-interests. Our mechanism also makes sure that information being shared among nodes is based on active participation of each node, avoiding the free-rider problem.

\section{EXPERIMENTAL EVALUATION AND RESULTS}

In this section we describe our simulated experimentation in detail taking into account the requirements for optimizing information dissemination using context-based grouping in a large scale network. Later on, we also discuss the results of our experiments and analyze the benefits. 


\section{A. Simulated Experimentation}

We have set up a simulation test bed to test our optimizing information dissemination using context-based grouping in a large scale peer-to-peer mobile network. We have used a realistic dataset to simulate 1300 cars. The dataset used for evaluation is a set of traces from the multi-agent microscopic traffic simulator (MMTS) ${ }^{1}$ developed by K. Nagel. It is capable of simulating traffic over real regional road maps of Switzerland with a high level of realism. The behavior of people is modeled and their movement with vehicles is reproduced for a period of 24 hours. Individuals in the simulation are distributed over cities and villages in an area of $250 \mathrm{~km} \times 260 \mathrm{~km}$. All individuals choose a time to travel and a route according to where they live and current road congestion. The complete dataset contains 260,000 vehicles with in total more than 25,000,000 recorded direction and speed changes. The parameters we have taken into account are for each node to perform simulated experiments;

$\begin{array}{ll}\text { (i) } & \text { Time } \\ \text { (ii) } & \text { Velocity } \\ \text { (iii) } & \text { Direction } \\ \text { (iv) } & \text { x and y Coordinates } \\ \text { (v) } & \text { Number of sent packets } \\ \text { (vi) } & \text { Number of received packets }\end{array}$

In our experiments, nodes move around like cars in virtual groups sharing same interests and let connections appear and disappear according to the communication range to other nodes. Some nodes acted as context providers whereas other nodes acted as context receivers. Relevance backpropagation algorithm is used for inter and intra-group communication. We divided the total area into sub-locations according to the physical communication characteristics of a wireless network. Dedicated Short Range Communication (DSRC) is commonly used for vehicle-to-vehicle and vehicle-toinfrastructure communication. It operates over $5.9 \mathrm{Ghz}$ band having a transmission range from $10 \mathrm{~m}-1000 \mathrm{~m}$ and tailored for high-speed mobile environments. We assumed the communication characteristics of DSRC in our experiments.

\section{B. Results and Analysis}

There are several types of messages in our simulated experimentation like (i) sent $\left(M_{s}\right)$, (ii) unique received $\left(M_{u r}\right)$, (iii) unique sent $\left(\mathrm{M}_{\mathrm{us}}\right)$, (iv) forwarded $\left(\mathrm{M}_{\mathrm{f}}\right)$, (v) duplicate $\left(\mathrm{M}_{\mathrm{d}}\right)$ and (vi) dropped $\left(\mathrm{M}_{\mathrm{drop}}\right)$. We measured the following parameters during our simulated experimentation and present results in Fig. 5.

Generally, broadcasting is currently being used in its various flavors (e.g. gradient broadcast, directed diffusion, multi-cast or two-tier dissemination) for information dissemination in large scale networks [16,18]. In the broadcasting scenario a vehicle shares information with all its peers without taking into account whether that information is of interest to them. In turn, each of these vehicles will again forward that information to their peers

1 http://www.lst.inf.ethz.ch/research/ad-hoc/car-traces/ until the time-to-live property of the message expires. This means that certain vehicles may receive the same information multiple times.

In the context-based grouping scenario the duplication of messages may also occur within a group but decreases significantly due to the use of our relevance backpropagation algorithm. Furthermore, context-based grouping will also eliminate the communication of irrelevant messages to nearby peers in the network more effectively by using context-based filtering.

Because we consider broadcasting to be the worst case scenario in terms of network efficiency we will relatively compare the improvement in terms of network traffic usage of the context-based grouping scenario. That is why network traffic usage with broadcasting has a $100 \%$ performance value. Regarding the relevancy of information we will measure how much worse broadcasting performs relatively to our context-based grouping mechanism. In this result contrary to the previous result - a lower value is worse.

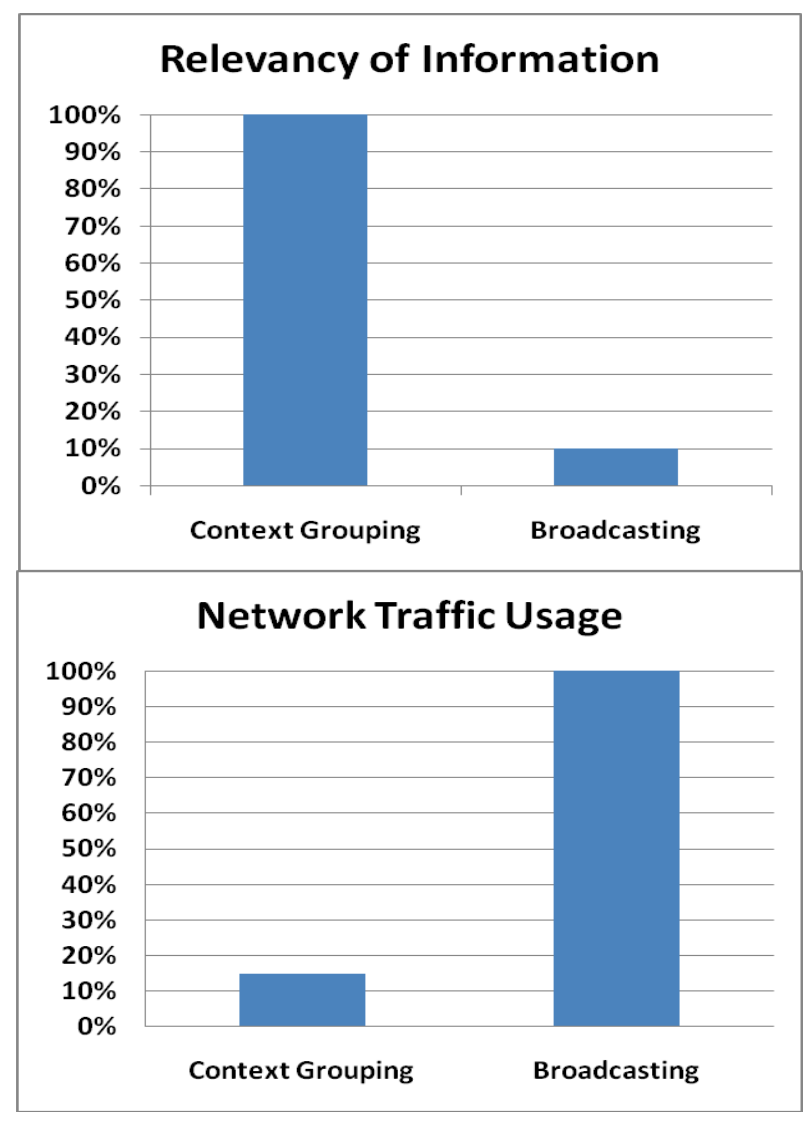

Fig. 5. Experimental results (relative) for our context-based grouping mechanism compared with plain broadcasting.

- $\quad$ Network Traffic (NT)

$$
\mathrm{NT}=\sum_{\mathrm{n}}\left(\mathrm{M}_{\mathrm{s}}+\mathrm{M}_{\mathrm{f}}\right)
$$

There is a significant difference in Network Traffic utilization of $85 \%$ for our context-based grouping mechanism in comparison to plain broadcasting as shown in Fig. 5 for this particular application. Again, lower network traffic utilization is considered to be better. 
The gain depends on the distribution of nodes across the geographical area of coverage, since a more uniform distribution means fewer nodes to communicate within each location. Moreover, the gain is also proportional to the average number of interests of a peer. Nonetheless, in other experiments with different applications we have gained similar improvements.

\section{- Relevancy $(R)$}

$$
\left.\mathrm{R}=\sum_{\mathrm{n}}\left(\left(\mathrm{M}_{\mathrm{ur}}+\mathrm{M}_{\mathrm{d}}\right)-\mathrm{M}_{\mathrm{drop}}\right)\right) / \sum_{\mathrm{n}}\left(\mathrm{M}_{\mathrm{ur}}+\mathrm{M}_{\mathrm{d}}\right)
$$

There is also a significant drop in the number of irrelevant message of about $90 \%$ in our context-based grouping mechanism compared to plain broadcasting. It illustrates that nodes in groups get more relevant information (i.e. the nodes receive less information they are not interested in) as shown in Fig. 5 for this particular application. Again, a higher relevancy percentage is considered to be better.

Referring back to the requirements discussed earlier, our context-based grouping with relevance backpropagation mechanism significantly lowers the communication overhead in terms of network traffic and optimizes delivery efficiency. While not explicitly shown in the results, our mechanism makes sure that the information is delivered to the right place at the right time effectively reducing the communication of irrelevant messages.

\section{RELATED WORK}

Optimizing context information dissemination is a vital characteristic for every context-aware application in use. In the literature, centralized solutions in which a server collects and manages information are numerous [3]. For example, Henricksen et al. [4] and Paganelli et al. [5], propose centralized entities, managing the context information and handling the client requests for it. These centralized approaches are prone to scalability and single point of failure. In our approach we optimize the information flow between the highly dynamic and mobile nodes in a large scale network using context-based grouping incorporated with our relevance backpropagation mechanism for information delivery only to the relevant nodes without relying on any centralized architecture.

Ye et al. [6] adopted a P2P approach for context sharing in which context information remains locally stored on the peers and only an access reference is registered on remote peers. The discovery of new peers is performed by broadcasting messages and context queries to remote peers are sent through unicast messages. Such a mechanism may raise scalability and security issues on ubiquitous environments as information is being disseminated to every known peer in the network. In our approach we use the relevance backpropagation algorithm to disseminate the right information at right place and time which helps group formation and group/node-to-group/node communication.

Peer-to-Peer communication supports high dynamic environments, with no need of centralized elements, providing a more flexible solution considering scalability and nodes failure $[7,8,11]$. The distributed aspects of the Peer-to-Peer pattern motivate the proposal of architectures such as [9], which implements a topic-based publish- subscribe system with SIP/SIMPLE [10]. Our approach is scalable to a significantly large sized network without relying on any centralized architecture and relevant information flow between and within the groups/peers in the network.

Cutting et al. [13] propose implicit group definition based on tags used by users to identify interesting content. Peers interested on the same content tags are considered to belong to the same implicit group. Messages are then addressed to tags forming the groups. Similarly Khambatti et al. [12] propose a grouping technique based on the notion of community. Communities are groups formed based on users (or peers) common interests, declared as peer attributes. Our approach is also stimulated by the dynamic group definition proposals presented earlier by these researchers. We improve this scheme by dividing groups based on interests, sub-interest and locations. The information flow is also intelligent and context-aware taking into the primary concerns of privacy by using our relevance backpropagation algorithm.

\section{CONCLUSION AND FUTURE WORK}

The Vehicles in a scalable environment may disseminate information about certain road traffic conditions, traffic incidents, free parking space or other relevant information to the neighboring vehicles in the vicinity. These vehicles form a large scale mobile peer-to-peer network in which information is shared among each other to perform contextsensitive tasks. However, traditional network addressing schemes are not well suited for group-based communication in large scale vehicular networks. The classical network paradigms of multicasting and broadcasting to define groups are too limited. First, there is no way to optimize network traffic based on the contextual characteristics of the nodes. Second, the groups of nodes are highly dynamic with vehicles randomly joining and leaving multiple groups. We propose an information dissemination approach based on context grouping in which only relevant information is shared among nodes. We evaluate our approach in a large scale vehicular network where groups are formed based on the location and shared interests of the nodes.

Our experimental results show that our context-based grouping mechanism significantly reduces the network traffic and ensures the right information is being disseminated to the right place and time. We can a significant difference in network traffic and relevancy of $85 \%$ and $90 \%$ respectively for our context-based grouping mechanism in comparison to plain broadcasting for a particular scenario as discussed earlier.

We plan to further investigate the network and context properties to get a broader view of the communication mechanisms used earlier for our experiments. We also plan to look into implicit grouping by analyzing network traffic and automatically inferring common interests of nodes without relying on the explicit definition of interests in an ontology. Moreover, we will couple our context-based grouping middleware with an existing simulation framework known as OMNET++, a real time discrete event-based 
network simulator. This will allows us to more easily analyze the effects of our algorithms and protocols for different scenarios and other mobile peer-to-peer application areas. This simulator will also help us to model the physical characteristics of vehicle networks which in turn will enable us to investigate the effects of non-received relevant messages. In the broadcasting scenario network channels are sometimes saturated with the effect that messages become irrelevant when they are finally delivered. In the contextbased grouping scenario filtering mechanisms may drop messages that turned out to be relevant for a particular peer. We cannot properly analyze this concern without incorporating the physical properties of an inter-vehicle network in our experiments.

\section{ACKNOWLEDGMENT}

This research is partially funded by the Interuniversity Attraction Poles Programme Belgian State, Belgian Science Policy, and by the Research Fund K.U.Leuven.

\section{REFERENCES}

[1] Behera, P. K. and Meher, P. K. (2002): Prospects of GroupBased Communication in Mobile Ad hoc Networks. In Proceedings of the 4th international Workshop on Distributed Computing, Mobile and Wireless Computing (December 28 31, 2002). S. K. Das and S. Bhattacharya, Eds. Lecture Notes In Computer Science, vol. 2571. Springer-Verlag, London, 174183.

[2] Kirsch-Pinheiro, M., Vanrompay, Y., Victor, K., Berbers, Y., Valla, M., Frà, C., Mamelli, A., Barone, P., Hu, X., Devlic, A., and Panagiotou, G. (2008): Context Grouping Mechanism for Context Distribution in Ubiquitous Environments. In Proceedings of the OTM 2008 Confederated international Conferences, Coopis, Doa, Gada, Is, and ODBASE 2008. Part I on on the Move To Meaningful internet Systems: (Monterrey, Mexico, November 09 - 14, 2008). R. Meersman and Z. Tari, Eds. Lecture Notes In Computer Science, vol. 5331. SpringerVerlag, Berlin, Heidelberg, 571-588.

[3] Baldauf, M., Dustdar, S., Rosenberg, F. (2007): A survey on context-aware systems. International Journal of Ad. Hoc. and Ubiquitous Computing 2(4), 263-277.

[4] Henricksen, K., Indulska, J., McFadden, T., Balasubramaniam, S. (2005): Middleware for distributed context-aware systems. In: International Symposium on Distributed Objects and Applications (DOA).

[5] Paganelli, F., Bianchi, G., Giuli, D. (2007): A Context Model for Context-Aware System Design Towards the Ambient Intelligence Vision: Experiences in the eTourism Domain. In: Stephanidis, C., Pieper, M. (eds.) ERCIM Ws UI4ALL 2006. LNCS, vol. 4397, pp. 173-191. Springer, Heidelberg.

[6] Ye, J., Li, J., Zhu, Z., Gu, X., Shi, H.: PCSM (2007): A Context Sharing Model in Peer-to-Peer Ubiquitous Computing Environment. In: International Conference on Convergence Information Technology, November 21-23, pp. 1868-1873.

[7] Delmastro, F., Passarella, A., Conti, M. (2008): P2P Multicast for pervasive ad-hoc networks. Pervasive and Mobile Computing 4(1), 62-91.

[8] Hu, X., Ding, Y., Paspallis, N., Bratskas, P., Papadopoulos, G.A., Barone, P., Mamelli, A.: A Peer-to-Peer based infrastructure for Context Distribution in Mobile and Ubiquitous Environments. In: Meersman, R., Tari, Z., Herrero, P. (2007): OTM-WS 2007, Part I. LNCS, vol. 4805, pp. 236-239. Springer, Heidelberg.
[9] Harjula, E., Ala-Kurikka, J., Howie, D., Ylianttila, M. (2006): Analysis of peer-to-peer SIP in a distributed mobile middleware system. In: IEEE Global Telecommunications Conference (Globecom 2006), pp. 1-6.

[10] Rosenberg, J. (2008): SIMPLE made Simple: An Overview of the IETF Specifications for InstantMessaging and Presence using the Session Initiation Protocol (SIP), IETF Draft, Expires August 27.

[11] Bryan, D., Matthews, P., Shim, E., Willis, D. (2008): Concepts and Terminology for Peer to Peer SIP, IEFT Draft, http://tools .ietf.org/html/draft-ietfp2psip- concepts-01 .

[12] Khambatti, M., Ryu, K.D., Dasgupta, P. (2004): Structuring peer-to-peer networks using interestbased communities. In: Aberer, K., Kalogeraki, V., Koubarakis, M. (eds.) VLDB 2003. LNCS, vol. 2944, pp. 48-63. Springer, Heidelberg.

[13] Cutting, D., Quigley, A., Landfeldt, B. (2007): Special Interest Messaging: A Comparison of IGM Approaches. The Computer Journal (Access published, December 2, 2007).

[14]Yasar, A., Preuveneers, D., and Berbers, Y. (2008): Adaptive context mediation in dynamic and large scale vehicular networks using relevance backpropagation. In Proceedings of the international Conference on Mobile Technology, Applications, and Systems (Yilan, Taiwan, September 10 - 12, 2008). Mobility '08. ACM, New York, NY, pp. 1-8.

[15] D. Preuveneers and Y. Berbers (2007): Architectural backpropagation support for managing ambiguous context in smart environments. In HCI (6), volume 4555 of Lecture Notes in Computer Science. Springer,.

[16] Mahajan, R., Zahorjan, J., and Zill, B. (2007): Understanding wifi-based connectivity from moving vehicles. In Proceedings of the 7th ACM SIGCOMM Conference on internet Measurement, IMC '07. ACM, New York, NY, 321-326.

[17] J. Indulska, T. McFadden, M. Kind, and K. Henricksen (2003): Scalable location management for context-aware systems. ACM.

[18] Leontiadis, I., Costa, P., and Mascolo, C. (2009): Persistent content-based information dissemination in hybrid vehicular networks. In Proceedings of the IEEE international Conference on Pervasive Computing and Communications (March 09 - 13, 2009). PERCOM. IEEE Computer Society, Washington, DC, 110. 